Original article

\title{
Thermal-induced hydrophilicity enhancement of titanium dental implant surfaces
}

\author{
Andrea Toffoli ${ }^{1}$, Ludovica Parisi $^{1}$, Roberta Tatti $^{2}$, Andrea Lorenzi ${ }^{3)}$, Roberto Verucchi ${ }^{4}$, Edoardo Manfredi ${ }^{1)}$, \\ Simone Lumetti ${ }^{1)}$, and Guido M. Macaluso ${ }^{1,4)}$
}

${ }^{1)}$ Dental School, Department of Medicine and Surgery, University of Parma, Parma, Italy

${ }^{2}$ Materials for Electronics and Magnetism Institute, National Research Council, Trento, Italy

${ }^{3)}$ Department of Chemical, Life and Environmental Sustainability Sciences, University of Parma, Parma, Italy

${ }^{4)}$ Materials for Electronics and Magnetism Institute, National Research Council, Trento, Italy

\begin{abstract}
Titanium surface characteristics, including microtopography, chemical composition, and wettability, are essential features to achieve osseointegration of dental implants, but the choice of a particular surface topography is still a debated topic among clinicians. An increased level of implant surface hydrophilicity has been demonstrated to ameliorate osseointegration and shorten healing times. The aim of this work is to develop and test a suitable thermal-based method to enhance titanium surface wettability without modifying other characteristics of the implant surface. For this function, titanium discs with different surface topography have been thermally treated by testing different temperatures and excluding those that led to evident chromatic and morphological modifications. The selected surface gain in wettability after the treatment was assessed through contact angle measurement, chemistry modifications through x-ray photoelectron spectroscopy (XPS) analysis, and microtopography through scanning electron microscopy (SEM). Results showed a great enhancement in hydrophilicity on the tested surfaces without any other modification in terms of surface chemical composition and topography. A possible limitation of this method could be the persistent, although relatively slow, biological aging of the surfaces after the treatment. The present findings indicate that the described treatment could be a safe and effective method to enhance dental titanium hydrophilicity and thus its biological performance.
\end{abstract}

Keywords; dental implant, hydrophilicity, scanning electron microscopy, titanium, $\mathrm{x}$-ray photoelectron spectroscopy

\section{Introduction}

Titanium has been long recognized to be the gold standard material for dental implants. To obtain clinical success, implants need to achieve osseointegration: a tight connection with bone without the interposition of fibrous tissue, which depends also on the characteristics of the implant surface, including microtopography, chemistry, and wettability $[1,2]$.

The effect of surface topography on primary osteoblasts' adhesion and proliferation, and consequently on osseointegration, has been studied for years. It is now well accepted that moderately rough implant surfaces elicit a better response when compared to a machined surface, enhancing primary stability and reducing the risk of early implant failure [3]. Conversely, machined implant surfaces show lower performances at early stages of implant placement but are $20 \%$ less likely to face the issue of periimplantitis [4]. Now that it has been established that different surface microtopography can influence short and long-term results of the implant therapy, the attention of researchers has moved to the amelioration of surface biological performances. Among other features, hydrophilicity influences the implant osseointegration rate in the first moments after its positioning. In particular, when the implant is soaked with blood, its wettability guides protein adsorption in terms of amount, strength of bonding, and tridimensional conformation [5]. More hydrophobic surfaces lead

Correspondence to Dr. Andrea Toffoli, Dental School, Department of Medicine and Surgery, University of Parma, Via Gramsci 14, Parma (PR) 43126, Italy

Email: andrea.toffoli@unipr.it

J-STAGE Advance Publication: March 11, 2020

Color figures can be viewed in the online issue at J-STAGE.

doi.org/10.2334/josnusd.19-0235

DN/JST.JSTAGE/josnusd/19-0235 to partial protein conformational changes, which may cause the loss of part of their function, thus leading to an ineffective and weak bonding to the surface. Moreover, this tridimensional modification of the protein structure could lead to the masking of cell-binding motifs, such as the arginine-glycine-aspartate (RGD) peptide, which are essential in developing a functional interaction between cells and the conditioned surface, potentially preventing an effective cell adhesion [6-8]. The effectiveness of super-hydrophilic surfaces has also been demonstrated in vivo, with hydrophilic implants showing a greater bone-to-implant contact 2-4 weeks after positioning than less hydrophilic ones [9-11]. Again, it has been demonstrated that hydrophilic surfaces positively regulate pathways directly involved in osteoblast differentiation and commitment, thus enhancing bone formation in the implant site [12].

Several methods have been recently developed to enhance titanium surface hydrophilicity, including photocatalysis, gamma ray and thermal treatment (TT) [13-15]. However, all these methods induce major modifications of other surface properties, such as microtopography changes, titanium dioxide thickness augmentation, and chemical composition changes $[13,16,17]$.

Furthermore, the biological aging of titanium, according to hydrophilicity and more generally, biological performances of titanium surfaces, tends to decrease after the processing procedures and is still an unsolved issue [18-21].

Considering these premises, the goal of the present work was to identify and test a TT that is able to enhance the wettability of titanium surfaces that leaves topographical, chemical and chromatic features unmodified and whose effects result long-lasting.

\section{Materials and Methods}

After testing an increasing range of temperatures to treat titanium, one target temperature was selected. Titanium discs treated with the target temperature were thus characterized physically through contact angle measurement, chemically by XPS, and morphologically by SEM.

\section{Titanium dises}

Commercially pure, grade 4 (ISO 5832/2) implant titanium discs with smooth machined or sand-blasted/acid-etched (SAE) surfaces and with a diameter of $8.0 \mathrm{~mm}$ and a thickness of $3.5 \mathrm{~mm}$ were used in this study. Discs were provided by Sweden \& Martina SpA. (Due Carrare, Padova, Italy) and were prepared with the manufacturing procedure of dental implants. Smooth machined samples were smooth surfaces not treated at the end of the milling process, whereas SAE samples were altered according to a proprietary protocol. All titanium discs were cleaned in an Argon-activated plasma reactor and sterilized prior to use, and their grade of wettability was low and stable in the previous month.

\section{Identification of the thermal treatment}

Different temperatures were tested to find the optimal thermal treatment (TT) that led to an evident gain of hydrophilicity without altering other surface properties. Potentially suitable TTs have been hypothesized starting from the treatment developed by MacDonald et al. [13]. For each temperature, discs were put into a programmable oven (Programat P310, Ivoclar Vivadent, Italy), heated from room temperature to the established temperature through a 1-hour heating-ramp, kept at the chosen temperature 


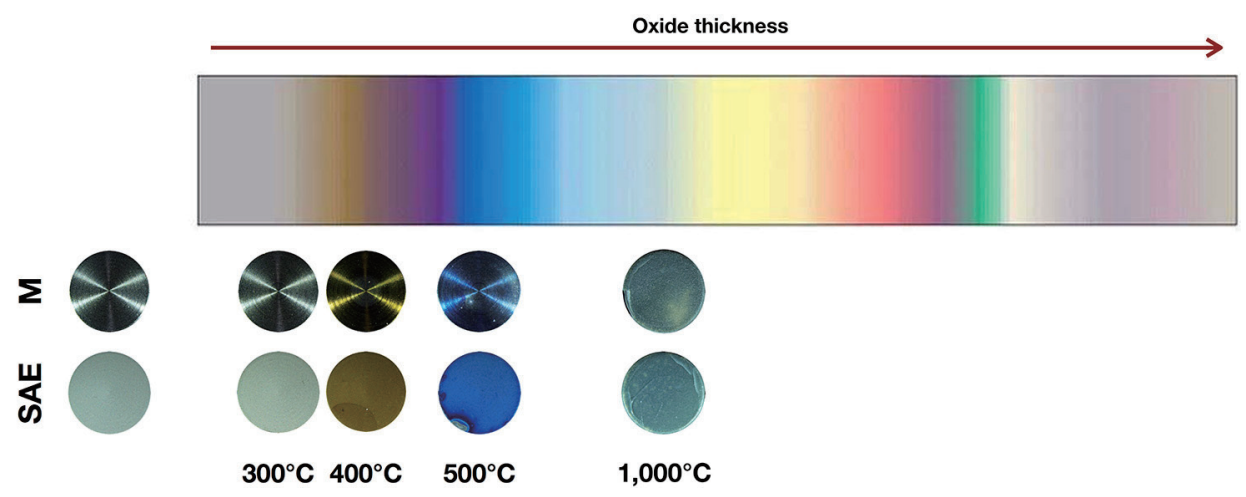

Fig. 1 Threshold temperature. Smooth machined and SAE samples compared to the color they assume after the exposition to different high temperatures. Chromatic change is a correlation of the augmentation of titanium dioxide thickness.
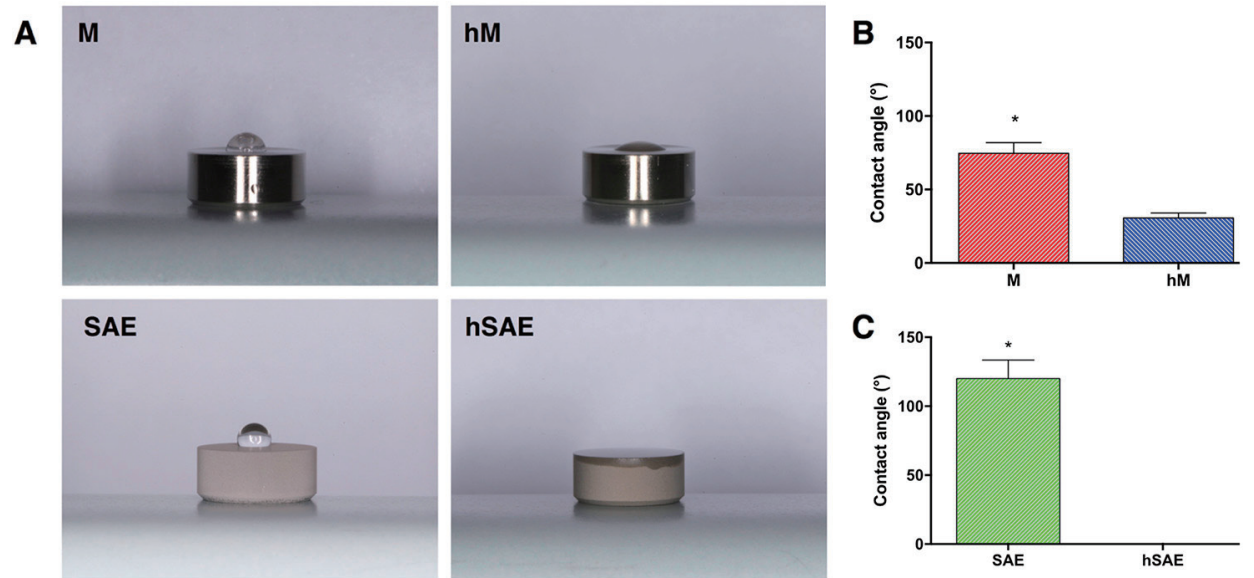

Fig. 2 Wettability test. (A) Standardized photographs representing the wettability of smooth machined and SAE titanium discs before and after the TT. (B-C) Histograms representing contact angle between smooth machined (B) or SAE (C) titanium surfaces and a $5 \mu \mathrm{L}$ water droplet before and after treatment. $* P<0.05$

for 1 hour, and finally, rapidly cooled down. Tested TT temperatures were $300^{\circ} \mathrm{C}, 400^{\circ} \mathrm{C}, 500^{\circ} \mathrm{C}$, and $1,000^{\circ} \mathrm{C}$.

Once an ideal TT to obtain hydrophilic surfaces was defined, discs were physically, chemically, and morphologically characterized.

\section{Wettability and short-term biological aging}

Differences in surface hydrophilicity were assessed by measuring the contact angles between the surfaces and a $5 \mu \mathrm{L}$ water droplet using 12 $\mathrm{SAE}$ and 12 smooth machined discs. The droplet was deposited on discs by using a pipette, and the contact angle with the surface was measured on standardized photographs through the ImageJ (ImageJ, US National Institutes of Health, Bethesda, MD, USA). Hydrophilicity was assessed before the treatment, immediately after the treatment, and then after 1, 2, $3,4,6,9,12,15,18$, and 24 days.

Data were analyzed using Prism 6 (GraphPad, La Jolla, CA, USA) and were reported as the mean \pm standard deviation of three repeated experiments performed in multiple replicates. The differences between groups were evaluated with non-parametric Kolmogorov-Smirnov statistical and considered significant when $P<0.05$.

\section{Chemical characterization}

XPS was used to investigate the differences in titanium surface chemistry. In particular, XPS analysis determined the chemical and electronic state of the elements on the surfaces. XPS was performed ex situ in an ultrahigh vacuum (UHV) apparatus, using the $\mathrm{Mg}-\mathrm{K} \alpha$ emission at $1253.6 \mathrm{eV}$ as $\mathrm{x}$-ray photon source. Photoelectrons were analyzed by a VSW HA100 electron energy analyzer, leading to a total energy resolution of $0.86 \mathrm{eV}$. Core level binding energies (BE) were referred to the $\mathrm{Au} 4 \mathrm{f} 7 / 2$ core level signal (at $84.0 \mathrm{eV}$ ), obtained from a sputtered gold surface. The photoemission core levels of all elements were analyzed through Voigt lineshape deconvolution after the background subtraction of a Shirley function. The typical precision for energy peak position was $\pm 0.05 \mathrm{eV}$, whereas the uncertainty for full width at half-maximum was less than $\pm 5 \%$ for area evaluation.

\section{Morphological characterization}

To observe any difference in the surface microtopography of titanium discs, samples were analyzed before and immediately after TT through SEM. This was observed using a dual beam Zeiss Auriga Compact system equipped with a GEMINI Field-Effect SEM column (Zeiss, Oberkochen, Germany) and the analysis was performed at $5 \mathrm{keV}$.

\section{Results}

\section{Thermal treatment}

All the TTs tested led to major and visible chromatic modifications due to the oxide layer thickening, with the exception of the treatment at $300^{\circ} \mathrm{C}$. At $400^{\circ} \mathrm{C}$, the TT changed the color of the sample to gold-yellow, to blue at $500^{\circ} \mathrm{C}$, and to gray-green at $1,000^{\circ} \mathrm{C}$ (Fig. 1). The color assumed by the discs was consistent with the color-scale based on oxide thickness. Moreover, although the chromatic aspect assumed by discs treated at $1,000^{\circ} \mathrm{C}$ was acceptable, the high temperature lead to a remarkable increase of the oxide thickness, which cancelled surface microtopography, making it impossible to distinguish smooth machined from SAE sample (Fig. 1).

Major modifications in surface color and in surface microtopography were the parameters used to discard higher temperature treatments, choosing $300^{\circ} \mathrm{C}$ as the target temperature to treat samples to obtain hydrophilic surfaces.

\section{Wettability and short-term biological aging}

The TT markedly increased titanium hydrophilicity. Histograms in Fig. 2 report the comparison of the contact angles on smooth machined and SAE samples, before and after TT. Even though the differences in hydrophilicity between treated and non-treated surfaces were statistically significant for both smooth machined (Fig. 2B: $P=0.0286$ ) and SAE (Fig. 2C: $P=$ 
Time-dependent hydrophilicity loss

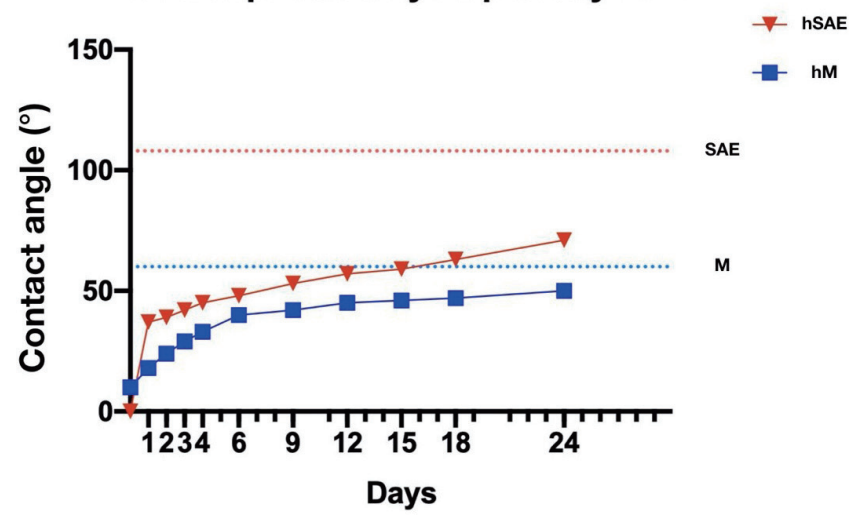

Fig. 3 Time-dependent hydrophilicity loss. Measurements of contact angle between a $5 \mu \mathrm{L}$ droplet and the treated surfaces after different periods of storage

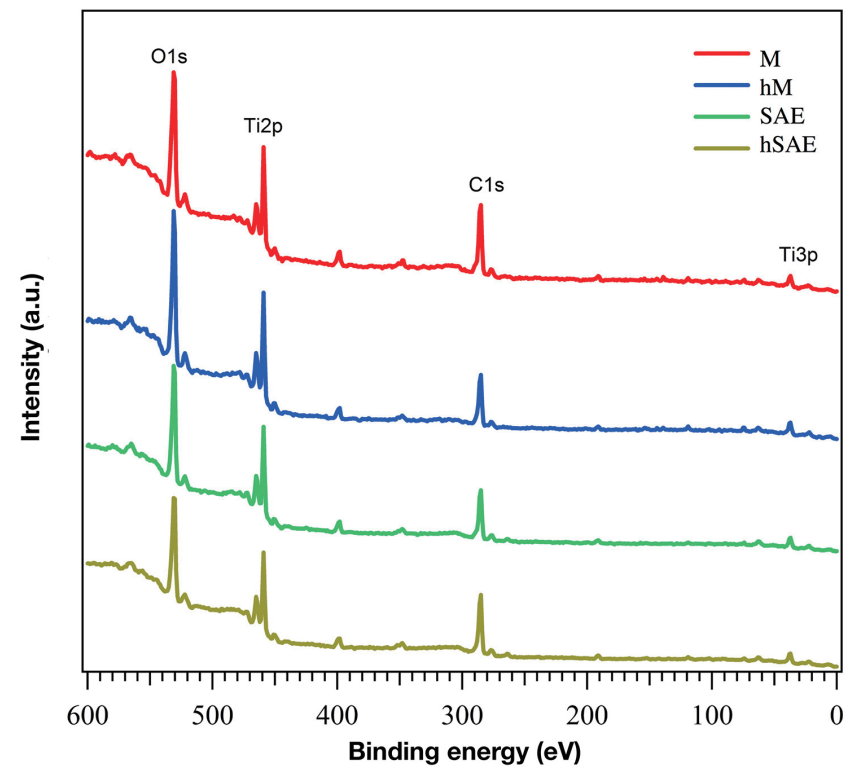

Fig. 4 Titanium surfaces chemical composition I. XPS wide range spectrum of the smooth machined or hM and of the SAE or hSAE titanium samples
A

\begin{tabular}{|c|c|c|c|c|}
\hline & M & hM & SAE & hSAE \\
\hline $\begin{array}{c}\text { Ti } \\
\text { (Titanium) }\end{array}$ & 9.47 & 10.98 & 11.04 & 9.25 \\
\hline $\begin{array}{c}\text { C } \\
\text { (Carbonium) }\end{array}$ & 44.68 & 37.63 & 42.26 & 48.64 \\
\hline $\begin{array}{c}\text { O } \\
\text { (Oxygen) }\end{array}$ & 35.97 & 40.00 & 37.24 & 34.04 \\
\hline other & 9.88 & 11.39 & 9.46 & 8.07 \\
\hline
\end{tabular}

B

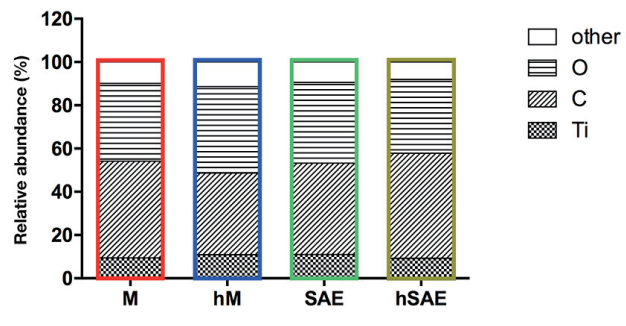

Fig. 5 Titanium surfaces chemical composition II. Table and histograms reporting the percentage contribution of titanium, carbon, oxygen, and other elements
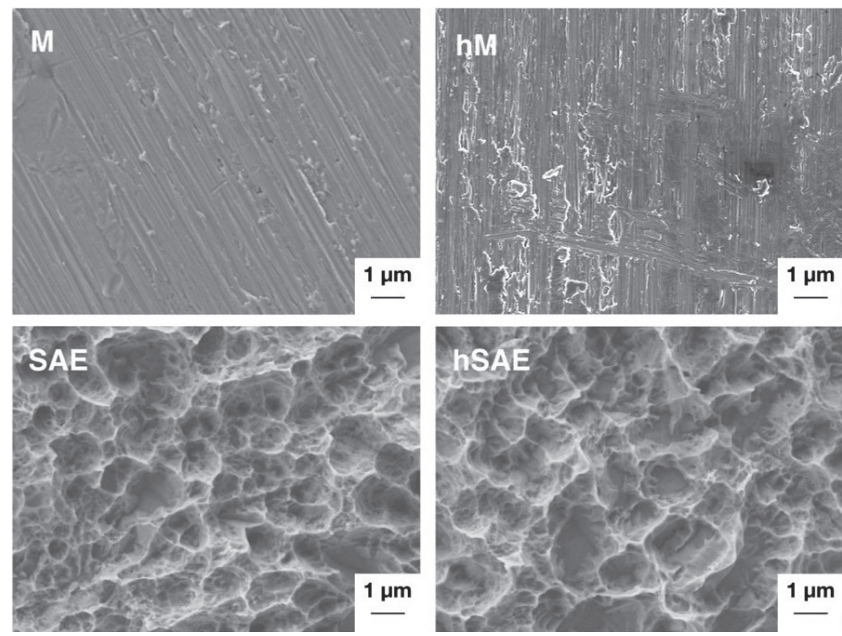

Fig. 6 SEM analysis. Typical SEM images of smooth machined, hM, SAE, and hSAE titanium surfaces

0.0286) surfaces, the gain in hydrophilicity was more marked for SAE than for smooth machined surfaces. Furthermore, this enhancement showed a time-dependent loss, even given that the differences between treated and non-treated surfaces were still evident 24 days after the treatment (Fig. 3).

\section{Chemical characterization}

XPS analysis revealed that the main chemical species discs, both before and after TT, were oxygen, titanium, and carbon, as shown in the wide range spectra reported in Fig. 4. In their oxidized chemical states, other minor contributions could be identified in a BE region between 30 and $150 \mathrm{eV}$. The chemical composition of all the samples is reported in Fig. 5 as surface atomic percentage. No significant differences were detected between smooth machined and SAE surfaces, as well as between normal and enhanced-hydrophilicity surfaces. The core level spectra of titanium (Ti2p) and oxygen (O1s) are reported in Fig. 7.

\section{Morphological characterization}

Regarding surface microtopography, smooth machined surfaces exhibited shallow parallel grooves that resulted from the manufacturing process, whereas SAE discs displayed a honeycomb pattern with cavities originating from sandblasting delimited by sharp ridges and constellated by smaller pits created by the etching process. SEM investigation revealed that TT did not alter the surface microtopography of titanium discs, and the morphological aspects of the surfaces were comparable for control groups and treated samples, either for smooth machined or for SAE surfaces (Fig. $6)$.

\section{Discussion}

It has been demonstrated that after dental implant positioning, surface microtopography, chemistry, and wettability play a major role in the short-term interaction with the surrounding biological milieu, as well as in implant osseointegration and reducing healing times [8]. Surface wettability is a fundamental characteristic involved in the obtaining of a biological stability because it promotes blood plasma protein adsorption and cell response [22,23]. In fact, it has been demonstrated that high implant surface hydrophilicity can lead to more a more efficient adsorption of pro- 
A

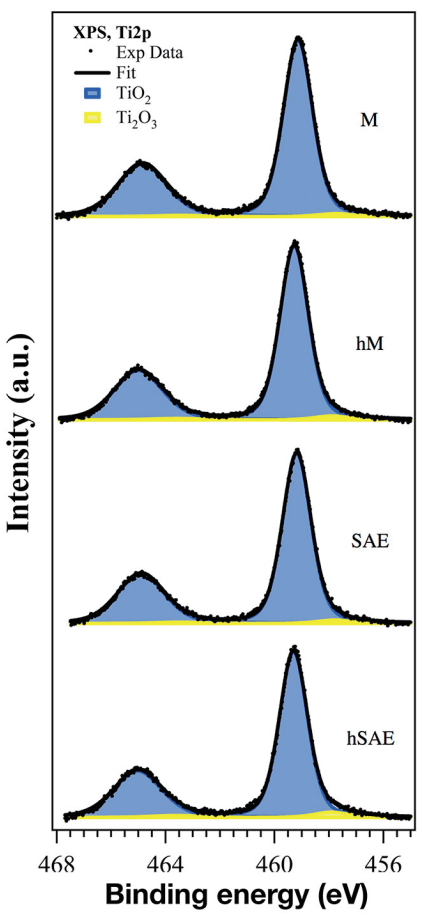

B

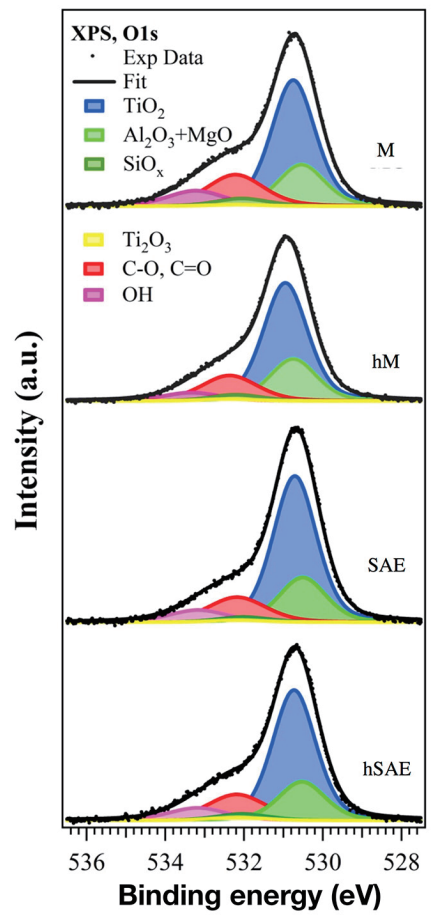

Fig. 7 XPS core level spectra (background subtracted). (A) Ti2p core level and (B) O1s core level. Experimental data plots (Exp Data) represent the total measured intensity. Titanium Ti2p core level was characterized by two main structures placed to a distance of $5.7 \mathrm{eV}$, as expected from the spin-orbit splitting of the $2 p$ peaks. From a chemical point of view, titanium was present in form of $\mathrm{TiO}_{2}\left(\mathrm{Ti}^{4+}\right)$ at 459.20 $\mathrm{eV}$, with a small contribution from $\mathrm{Ti}_{2} \mathrm{O}_{3}$, located at lower binding energy $(457.80 \mathrm{eV})$. O1s core level analysis was more complex, due to the presence of six components correlated with all the oxide species of carbon, titanium, magnesium, aluminum, and silicon. Main peaks at $530.80 \mathrm{eV}$ refer to oxygen in $\mathrm{TiO}_{2}$ (blue component), while different species from $\mathrm{Al}_{2} \mathrm{O}_{3}$ and $\mathrm{MgO}$ could not be resolved and gave rise to component at $530.60 \mathrm{eV}$ (light green component). Other components were present, attributable to $\mathrm{C}=\mathrm{O}$ carbonyl groups at $532.25 \mathrm{eV}$ (red component) and hydroxyl groups on the titanium oxide at $533.20 \mathrm{eV}$ (pink component), and other minor contributions coming from silicon oxides at $532.10 \mathrm{eV}$ (dark green component) and $\mathrm{Ti}_{2} \mathrm{O}_{3}$ at $532.20 \mathrm{eV}$ (yellow component) were detected as well.

teins from biological fluids (e.g. blood plasma), which grants a functional surface-cell adhesion [6-8].

Recently, different methods have been developed to obtain hydrophilic surfaces; however, no effective TTs have been found to ameliorate titanium surface wettability while leaving microtopography, color, and chemical composition unmodified.

Different temperatures have been tested to obtain hydrophilic surfaces, and the $300^{\circ} \mathrm{C}$ treatment has been selected as the target temperature (Fig. 1). Although the $1,000^{\circ} \mathrm{C}$ treatment showed an acceptable chromatic aspect of the discs, this temperature treatment was not considered, as the dioxide thickness increase was so evident that is was impossible to recognize smooth machined from SAE surface profiles.

Surface contact angle measurement showed that the selected temperature was effective in guaranteeing a major increase in surface hydrophilicity, especially on SAE surfaces where the droplet completely flattened. Different surface textures influence hydrophilicity: Smooth machined samples have higher surface hydrophilicity than SAE samples, but the TT shows more efficacy on the SAE samples, leading those surfaces to the maximum grade of hydrophilicity (Fig. 2).

To explain this, a mild oxidation of the surfaces following the TT [13] and consequently an increase of the oxygen percentage of the surface were expected, but XPS analysis showed no significant differences in terms of chemical composition (Figs. 4 and 5). It is believable to think that this finding is due to the sampling depth of XPS analysis $(-10 \mathrm{~nm})$, which is limited to the outermost surface and also to the low temperature of the treatment. Consistently with this, the thickening of the oxide layer was so slight that both SEM and atomic force microscope showed no modifications in terms of surface topography.

According to this theory, Lee et al. studied the surface modification on thermal-treated, smooth, commercially-pure titanium surfaces and found that higher temperatures used to obtain hydrophilic surfaces led to major and evident surface modifications in terms of titanium dioxide thickness and roughness modifications [17]. Moreover, MacDonald et al. studied the effects of chemical and heating treatments on TiAlV6 alloy surfaces, which are mainly used for orthopedic prosthesis, observing that TTs with a temperature higher than $400^{\circ} \mathrm{C}$ increased the alloy surface hydrophilicity and modified its microtopography $[13,17]$. Furthermore, it is known from the literature that titanium dioxide thickness faces slight modifications if titanium is heated to a temperature lower than $400^{\circ} \mathrm{C}$ [17]. It has been demonstrated that the gain in titanium dioxide thickness causes the change in surface color, which is not acceptable for esthetic reasons [16].

Ogawa et al. presented a different protocol based on the UV-functionalization of titanium surfaces. They showed that UV-functionalized titanium surfaces present a titanium dioxide layer with a positive electrostatic charge, which they showed to be the key effect of this functionalization in creating a direct interaction between titanium and proteins and also between titanium and osteoblasts, superseding the effect of hydrophilic status $[24,25]$. However surface hydrophilicity-driven key protein conformation $[7,8]$ is still a fundamental aspect to be considered when observing protein-mediated cell behavior on implant surfaces.

Consistently with the present literature, the gain in hydrophilicity obtained through the described method has been shown to progressively decrease over time, even if it was still present after 24 days (Fig. 3). In the perspective of a future clinical application, this fact makes it useless to add the TT in the implant production process, making it necessary to perform the TT as a "chairside" procedure, possibly through a TT-resistant packaging. Another possible solution to be tested is developing a packaging able to avoid issues related to titanium biological aging.

In conclusion, it is possible to believe that the TT described in this work is a viable method to obtain hydrophilic surfaces. Further experiments will be now necessary to test the efficiency of the hydrophilic surfaces to trigger the proper sequence of biological events on which dental implant clinical success relies. 


\section{Acknowledgments}

The authors would like to thank Sweden \& Martina SpA. for titanium discs manufacturing and providing, as well as Dr. Enrico Babetto and Dr. Silvia Cuccarolo for their precious advice. Dr. Ludovica Parisi received support from a research scholarship of the Osteology Foundation (Lucerne, Switzerland) and from the Fondazione Cariparma (Parma, Italy).

\section{Conflict of interest}

The authors have no conflict of interest to disclose.

\section{References}

1. Abrahamsson I, Berglundh, T, Linder E, Lang NP, Lindhe J (2008) Early bone formation adjacent to rough and turned endosseous implant surfaces. Clin Oral Implant Res 15, 381392.

2. Lang NP, Salvi GE, Huynh-Ba G, Ivanovki S, Donos N, Bosshardt DD (2011) Early osseointegration to hydrophilic and hydrophobic implant surfaces in humans. Clin Oral Implant Res 22, 349-356.

3. Ivanoff C, Hallgren C, Widmark G, Sennerby L, Wennerberg A (2001) Histologic evaluation of the bone integration of TiO (2) blasted and turned titanium microimplants in humans. Clin Oral Implant Res 12, 128-134.

4. Esposito M, Ardebili Y, Worthington H (2014) Interventions for replacing missing teeth: different types of dental implants. Cochrane Database Syst Rev 7, CD003815.

5. Calciolari E, Mardas N, Dereka X, Anagnostopus A, Tsangaris G, Donos N (2018) Protein expression during early stages of bone regeneration under hydrophobic and hydrophilic titanium domes. A pilot study. J Periodontal Res 53, 174-187.

6. Wilson CJ, Clegg RE, Leavesley DI, Pearcy MJ (2005) Mediation of biomaterial - cell interactions by adsorbed proteins: a review. Tissue Eng 11, 1-18.

7. Vogler E (2012) Protein adsorption in three dimensions. Biomaterials 33, 1201-1237.

8. Gittens R, Scheideler L, Rupp F, Hyzy S, Geis-Gerstorfer J, Schwartz Z (2014) A review on the wettability of dental implant surfaces II: biological and clinical aspects. Acta Biomater 10, 2907-2918.

9. Buser D, Broggini N, Wieland M, Schenk R, Denzer A, Cochran D (2004) Enhanced bone apposition to a chemically modified SLA titanium surface. J Dent Res 83, 529-533.

10. Lang N, Salvi G, Huynh-Ba G, Ivanovski S, Donos N, Bosshardt D (2011) Early osseointegration to hydrophilic and hydrophobic implant surfaces in humans. Clin Oral Implant Res 22, 349-356.

11. Donos N, Horvath A, Mezzomo L, Dedi D, Calciolari E, Mardas N (2017) The role of immediate provisional restorations on implants with a hydrophilic surface: a randomised, single-bin controlled clinical trial. Clin Oral Implant Res 29, 55-66.

12. Calciolari E, Hamlet S, Ivanoski S, Donos N (2018) Pro-osteogenic properties of hydrophilic and hydrophobic titanium surfaces: crosstalk between signalling pathways in in vivo models. J Periodont Res 53, 598-609.

13. MacDonald D, Rapuano B, Deo N, Stranick M, Somasundaran P, Boskey A (2004) Thermal and chemical modification of titanium-aluminum-vanadium implant materials: effects on surface properties, glycoprotein adsorption, and MG63 cell attachment. Biomaterials 25 , 3135-3146.

14. Aita H, Hori N, Takeuchi M, Suzuki T, Yamada M, Anpo M (2009) The effect of ultraviolet functionalization of titanium on integration with bone. Biomaterials 30, 1015-1025.

15. Ueno T, Takeuchi M, Hori N, Iwasa F, Minamikawa H, Igarashi Y (2012) Gamma ray treatment enhances bioactivity and osseointegration capability of titanium. J Biomed Mater Res B Appl Biomater 100, 2279-2287.

16. Neupane M, Kim Y, Park I, Lee M (2008) Characterization of surface oxide films and cell toxicity evaluations with a quenched titanium surface. Met Mat Intern 14, 443-448.

17. Lee Y, Cui D, Jeon H, Chung H, Park Y, Kim O (2012) Surface characteristics of thermally treated titanium surfaces. J Periodontal Implant Sci 42, 81-87.

18. Att W, Hori N, Takeuchi N, Ouyang J, Yang Y, Anpo M et al. (2009) Time-dependent degradation of titanium osteoconductivity: an implication of biological aging of implant materials. Biomaterials 30, 5352-5363.

19. Lee JH, Ogawa T (2012) The biological aging of titanium implants. Implant Dent 2, 415421.

20. Minamikawa H, Att, W, Ikeda T, Makoto H, Ogawa T (2016) Long- term progressive degradation of the biological capability of titanium. Materials $9,102$.

21. Manfredi E, Lumetti S, Rivara F, Toffoli A, Calciolari E, Cacchioli A et al. (2016) Role of prostaglandin E2 in the modulation of Wnt canonical signaling in cells on microstructured titanium surfaces. J Appl Biomater Funct Mater 14, 181-188.

22. Eriksson $\mathrm{C}$, Nygren $\mathrm{H}$, Ohlson $\mathrm{K}$ (2004) Implantation of hydrophilic and hydrophobic titanium discs in rat tibia: cellular reactions on the surfaces during the first 3 weeks in bone. Biomaterials 25, 4759-4766.

23. Bornstein M, Valderrama P, Jones A, Wilson T, Seibl R, Cochran D (2008) Bone apposition around two different sandblasted and acid-etched titanium implant surfaces: a histomorphometric study in canine mandibles. Clin Oral Implant Res 19, 233-241.

24. Iwasa F, Hori N, Ueno T, Minamikawa H, Yamada M, Ogawa T (2010) Enhancement of osteoblast adhesion to UV-photofunctionalized titanium via an electrostatic mechanism. Biomaterials 31, 2717-2727.

25. Hori N, Ueno T, Minamikawa H, Iwasa F, Yoshino F, Kimoto K et al. (2010) Electrostatic control of protein adsorption on UV-photofunctionalized titanium. Acta Biomaterialia 6, 4175-4180. 